\title{
然
}

José Luis Káiser Moreiras*

\section{PLAN CHINA DE LA SECRETARÍA DE ESTADO DE COMERCIO}

El Plan China es el primer plan PASE (País con Actuación Sectorial Estratégica) que se está elaborando para consolidar, incrementar y diversificar la exportación española, todo ello en el marco de la Estrategia de Internacionalización de la Economía Española 2017-2027 (EIEE), que fue aprobada en el Consejo de Ministros del pasado 8 de septiembre. La EIEE surge bajo el nuevo modelo de desarrollo sostenible de la economía española, que requiere asegurar la contribución positiva estructural del sector exterior con un impacto positivo sobre la creación de empleo. De ahí la necesidad de una estrategia de internacionalización a medio plazo para cohesionar todas las acciones en materia de internacionalización de los diferentes ministerios y comunidades autónomas. La Secretaría de Estado de Comercio ha querido empezar por el plan PASE para China por la importancia del continente asiático en la economía mundial y estar considerado como un país prioritario en sus políticas de internacionalización. Con China tenemos una cuota muy inferior a la nuestra en el comercio mundial y estamos proporcionalmente más lejos que nuestros competidores. Podemos afirmar que sin un crecimiento sostenido de nuestras exportaciones al mercado chino va a ser difícil incrementar nuestra cuota en los mercados mundiales en los próximos años. Actualmente, el saldo negativo de nuestro comercio con China es prácticamente igual a la totalidad del déficit comercial español.

Palabras clave: internacionalización, economía española, competitividad, comercio internacional, inversiones directas extranjeras (IDE), empresas españolas exportadoras, sectores prioritarios de exportación, países prioritarios para exportar.

Clasificación JEL: F13, F21, F43, O19, O53.

\section{Introducción}

El Plan China es el primer PASE (País con Actuación Sectorial Estratégica) que se va a lanzar en el marco de la Estrategia para la Internacionalización de la Economía Española

\footnotetext{
*Director General de Comercio Internacional e Inversiones. Secretaría de Estado de Comercio.

Versión de enero de 2018.
}

2017-2027 (EIEE). Este plan será un ejemplo y modelo a seguir para la redacción de los futuros planes en otros países prioritarios.

Como comentaré más adelante, los países PASE vienen a reemplazar a los PIDM (Planes Integrales de Desarrollo de Mercado), que fueron lanzados en 2005 y que con el tiempo llegaron a ser 16 países o regiones sobre los que se desarrollaron estos planes. El objetivo de los $\triangleright$ 
PASE, como en su momento fueron los PIDM, es diversificar la oferta exportadora española e intensificar los intercambios económicos y comerciales en mercados con alto potencial de crecimiento fuera de la Unión Europea. No podemos olvidar que todavía aproximadamente dos terceras partes de nuestras exportaciones de mercancías van dirigidas a la UE-28, lo que pone de manifiesto una relativa concentración de nuestras exportaciones en el Mercado Único. En el siguiente apartado veremos el salto cualitativo que supone este cambio de enfoque desde los PIDM hacia los PASE.

En el marco de este nuevo enfoque, en la Secretaría de Estado de Comercio hemos optado por empezar a diseñar los planes de los países del mercado asiático por dos motivos fundamentales: por un lado, a nadie se le escapa el peso económico de la región (segundo continente por valor de sus importaciones, tanto en bienes como en servicios) y sus buenas perspectivas de crecimiento en los próximos años; y por el otro, España, a pesar de los esfuerzos realizados a todos los niveles, tanto públicos como privados, todavía tiene una presencia pequeña en esta región (solamente el 0,5 por 100 de las importaciones de Asia proceden de España, en relación a una cuota del 1,8 por 100 a nivel mundial). A este hecho hay que añadir que Asia (excluyendo Oriente Medio) es con diferencia el área con la que España registra el mayor déficit: 30.634 millones de euros en 2016, casi $2 / 3$ partes del mismo con China (18.820 millones).

Sin embargo, en el caso de China tenemos que tener en cuenta de dónde veníamos y cómo hemos avanzado en los últimos tres lustros. Por poner algún ejemplo ilustrativo, del año 2000 al 2016 la exportación de España a China se ha multiplicado casi por diez y en el periodo 2006-2016, casi tres veces hasta los
5.031 millones de euros. En el periodo 20102016 el número de empresas españolas exportadoras a China ha pasado de las 6.586 hasta las 15.051 , a pesar de la complejidad y fuerte competencia existente.

Si nos referimos a los flujos de inversión, la inversión neta de China a España ha sido casi inexistente hasta el año 2011 , siendo especialmente importante en los años 2015 y 2016 , con 1.483 y 1.117 millones de euros respectivamente. En el año 2015 (último año disponible), la posición o stock de la inversión de China en España alcanzó los 9.206 millones de euros en operaciones no ETVE1.

En cuanto a los flujos de inversión de empresas españolas a China, esta ha tenido una evolución más irregular, con un pico en el año 2010 (1.303 millones de euros de inversión neta no ETVE), y todavía es relativamente pequeña en relación al stock mantenido en el resto del mundo (la posición de la inversión española no ETVE en China era de 2.769 millones de euros en 2015 o apenas el 1 por 100 del total del stock de España en el exterior). Hubo cierta avalancha de empresas españolas en el mercado chino en un momento dado (2010-2011) y después, ante cierta decepción en el cumplimiento de las expectativas empresariales, dichos flujos se han contraído en los últimos años. A eso hay que sumarle una disminución generalizada de la inversión española en países emergentes y países en vías de desarrollo en los últimos años.

Por otro lado, las relaciones institucionales entre España y el Gobierno chino siguen siendo excelentes, como lo muestran las numerosas visitas y viajes institucionales que se realizan anualmente entre ambos países. El 6 de noviembre de 2017 se celebró en Madrid la $\triangleright$

Entidades de tenencia de valores extranjeros, por lo que la inversión no ETVE excluye estas sociedades instrumentales. 
XXVII Comisión Mixta Anual de Cooperación Económica e Industrial Hispano-China. La copresidieron por parte China el viceministro de Comercio, Ziying Fu, y, por parte de España, la secretaria de Estado de Comercio, María Luisa Poncela. En dicha comisión, y en un ambiente de máxima colaboración y de buen entendimiento a nivel político, se trataron los distintos temas que interesan a ambos países para reforzar e incrementar sus relaciones económicas y comerciales bilaterales.

Se comprobó que son muchos los temas, tanto a nivel bilateral como multilateral, sobre los que se requiere seguir trabajando para facilitar la expansión y diversificación de las relaciones económicas y comerciales entre China y España. A corto plazo, se espera que las cifras de exportación continúen mejorando y que la puesta en vigor del Convenio Bilateral de Seguridad Social, así como una rápida actualización del Convenio de Doble Imposición, dé un balón de oxígeno que necesitan nuestras empresas para incrementar los flujos de inversión en ambas direcciones. Asimismo, el Grupo de Trabajo de Inversiones ayudará a resolver problemas de inversión de empresas españolas y chinas. Otra línea de trabajo que se quiere fortalecer es la cooperación en terceros mercados que permitirá identificar proyectos conjuntos a desarrollar en América Latina, África y Asia.

En definitiva, el Gobierno de España sigue apostando fuerte por el mercado asiático, empezando por el gigante chino, si bien con posterioridad se aprobarán los PASE para otros países de la región (Japón, Corea del Sur, India y Singapur). Sabemos que la distancia geográfica y las barreras culturales y lingüísticas son un obstáculo para nuestras empresas, pero precisamente por eso nuestros esfuerzos junto con el sector privado se han de redoblar en esta área tan dinámica.

\section{De los PIDM a los PASE}

El Consejo de Ministros aprobó, el 8 de septiembre de 2017, la Estrategia de Internacionalización de la Economía Española 2017-2027 (EIEE), que venía a reemplazar al Plan Estratégico de la Internacionalización de la Economía Española 2014-2015 (PEIEE). En este plan ya se recogían prioridades geográficas y por primera vez se establecían prioridades sectoriales. Sin embargo, era necesario tener una visión a medio plazo del papel a desarrollar por el sector exterior en el crecimiento sostenible de la economía española, en un marco de mayor coordinación institucional, tanto a nivel público como privado.

La EIEE 2017-2027 se desarrollará mediante planes de acción bienales, siendo el primero de ellos para el periodo 2017-2018. Los planes de acción incorporarán un conjunto de medidas concretas que el Gobierno busca poner en marcha para apoyar a los exportadores y atraer inversión extranjera hacia España. En dicha estrategia se decidió también reestructurar los PIDM² (Planes Integrales de Desarrollo de Mercado), dándoles un enfoque de carácter más sectorial y añadiendo algunos aspectos como el refuerzo en la coordinación institucional, la implementación de nuevos instrumentos de apoyo a la internacionalización o dar mayor énfasis a la evaluación y al seguimiento. Este nuevo enfoque son los planes PASE. La elección de los países PASE se realizó basándose en un análisis cuantitativo de una serie de indicadores objetivos sobre el potencial de los mercados para las empresas y $\triangleright$

2 Desde 2005 estaban operativos los PIDM, que se fueron perfeccionando y ampliando hasta contar entonces con 16 países o regiones sobre los que se desarrollaron estos planes. Los PIDM eran Argelia, Australia, Brasil, China, Países del Consejo de Cooperación para Ios Estados Árabes del Golfo, Corea del Sur, EEUU, India, Indonesia, Japón, Marruecos, México, Rusia, Singapur, Sudáfrica y Turquía. 
con la información proporcionada por la red de Oficinas Económicas y Comerciales de España (Ofecomes) y por el ICEX.

En una primera fase, los países seleccionados como PASE son trece: Estados Unidos, China, México, Singapur, India, Marruecos, Canadá, Japón, Corea del Sur, Brasil, Turquía, Rusia y Sudáfrica. Para cada uno de ellos se ha seleccionado una serie de sectores estratégicos con base en un análisis cuantitativo en los que centrar las actividades coordinadas de apoyo a su internacionalización. Está previsto a corto plazo incorporar el sector privado en la definición de esta nueva estrategia PASE. En ningún caso se trata de una lista excluyente ni cerrada, es decir, no se van a dejar de realizar actuaciones determinadas en apoyo de la internacionalización en otros sectores y países no incluidos.

Tanto en los PIDM como en los PASE rige «el principio de adicionalidad de la actuación pública», esto es, el empleo eficiente de los recursos y, por tanto, la necesidad de dar prioridad desde el punto de vista geográfico ( $\mathrm{y}$ sectorial en el caso de los PASE) a aquellos mercados más atractivos para el conjunto de la oferta española fuera de nuestro mercado natural de la UE. En el caso de los PASE, se seleccionan los sectores de oportunidad en determinados mercados que mejor encajan con la oferta exportable y donde las empresas españolas cuentan con un amplio margen de mejora para consolidar su presencia exterior. En la selección de estos sectores, además del sector privado, tienen protagonismo las Ofecomes, por tener información de primera mano sobre las oportunidades que se abren a las empresas españolas en los distintos mercados.

Tal y como comentaba en la introducción, existe un salto cualitativo en el nuevo enfoque de los PASE respecto a los PIDM y no se trata solamente de introducir el ámbito sectorial. Ante la creciente competencia en el mundo globalizado donde vivimos, a las instituciones españolas nos toca ser más creativas y tener estrategias más sofisticadas para poder seguir siendo competitivas. Los PIDM jugaron un papel importante en su momento porque permitieron planificar todas las medidas institucionales, financieras, de promoción y formación que se llevaban a cabo en los siguientes dos años de su aprobación para incrementar nuestras relaciones económicas y comerciales con los países y regiones elegidos. Este ejercicio permitía que hubiera una mayor coherencia entre las actuaciones de los distintos organismos de apoyo oficial a la exportación e inversión.

Sin embargo, los PASE van más lejos en este ejercicio que los PIDM, ya que aquellos introducen un componente de «inteligencia económica», esto es, un análisis que permite ver qué sectores de oportunidad de los países mencionados encaja con la oferta exportadora española. Asimismo, en los PASE se introducen los seis ejes estratégicos del EIEE 2017-2027 que engloban los aspectos más importantes de la internacionalización de una economía: buscar un apoyo a la empresa más adaptado a su perfil y necesidades, incorporar la innovación, tecnología y digitalización a la internacionalización, ampliar la formación de estudiantes y profesionales, aprovechar mejor la acciones de política comercial, tanto a nivel bilateral como comunitario y multilateral, y reforzar una mayor coordinación público-privada e institucional, tanto interministerial como con las comunidades autónomas (CCAA).

Como no podría ser de otra forma, la parte fundamental de los PASE serán las actividades que van a realizar a lo largo del año, las cuales se revisarán con una periodicidad anual. Como veremos en el Plan China, estas actividades $\triangleright$ 
incluirán medidas que se han venido aplicando en los últimos años, pero incluiremos otras tantas innovadoras que tendrán encaje en los ejes estratégicos de la EIEE y, en algunos casos, estarán asignadas a los sectores prioritarios.

Asimismo, la EIEE 2017-2027 incorpora un ejercicio de evaluación que permite medir los resultados de los sucesivos planes de actuación bienales y realizar un seguimiento pormenorizado del cumplimiento de los objetivos fijados. Para eso se seleccionará una serie de indicadores que sean estratégicos (dentro de los que ya están contemplados en el Plan de Acción) como otros que midan las actuaciones de la Administración Pública.

\section{Importancia de Asia: por qué China}

\subsection{Comercio de bienes de España por grandes áreas geográficas}

Asia fue en 2016 el tercer destino de las exportaciones españolas, tras la UE-28 y África, con el 6,1 por 100 , y el segundo origen de las importaciones con el 16,9 por 100, por detrás de la UE-28. Asimismo, en el periodo 2000-2016, ha sido la segunda área tras África en aumento de peso de las exportaciones, con 2,5 puntos porcentuales, $y$ la primera con respecto a las importaciones, con 6,3 puntos porcentuales de ganancia.

Asia es el segundo continente por valor de importaciones mundiales de bienes, con el 30,3 por 100 de las mismas en 2016, tan solo por detrás del mercado europeo (37,5 por 100). China es el segundo mercado de importación mundial, con casi el 10 por 100 del total de las importaciones mundiales, solo por detrás de EEUU (13,9 por 100). A su vez, en servicios comerciales, Asia es el segundo continente por valor de importaciones de servicios del mundo, con el 30,5 por 100 de las mismas en 2016, tan solo por detrás del mercado europeo (42,9 por 100). China es también el segundo mercado de importación mundial, con un porcentaje muy similar $(9,6$ por 100), por detrás nuevamente de EEUU (10,4 por 100).

EI FMI prevé que los países del ASEAN-5 lideren el crecimiento de las importaciones tanto de bienes como de bienes y servicios hasta el año 2022 (45,7 por 100 en el periodo 20172022). El crecimiento de las importaciones de China (26,8 por 100) será inferior al del conjunto de estos países, pero superior al de las economías avanzadas (25 por 100).

\subsection{Exportaciones españolas}

De los 25 principales destinos de las exportaciones españolas en 2016, 13 son países de la UE-28, por tan solo 2 asiáticos, con China en la 11. ${ }^{a}$ posición, que aglutinó tan solo el 2 por 100 (pero 1,5 puntos porcentuales más que en el año 2000), y Japón en la 16. a , con el 0,9 por 100, menos de una décima que en 2000.

Con respecto a los clientes con mayor dinamismo en cuanto a ganancia de peso e incremento de las importaciones procedentes de España con respecto al año 2000, destacan Marruecos y China, con una ganancia de 1,6 y 1,5 puntos porcentuales (pp) respectivamente, seguidos por Polonia (1,1 pp), Argelia (0,7 pp) y Rumanía (0,7 pp). Por su parte, Japón, el otro país asiático de los 25 principales países, mantuvo su peso estable.

\subsection{Importaciones españolas}

De los 25 principales orígenes de las importaciones españolas, 11 son países que $D$ 
pertenecen a la UE-28, destacando Alemania y Francia por encima del resto, que acumulan un cuarto de las importaciones españolas. Con respecto a los países asiáticos, en este caso hay cuatro: China, Japón, India y Vietnam. Precisamente, el primer proveedor de fuera de la UE y tercero en el ranking general es China, con el 8,7 por 100 en 2016. A gran distancia se sitúan Japón e India, con el 1,3 por 100 cada uno, y Vietnam, con el 0,9 por 100.

Con respecto a los proveedores con mayor dinamismo, en cuanto a ganancia de peso e incremento de las importaciones con respecto al año 2000, destaca sin parangón China, con una ganancia de casi 6 pp con respecto al año 2000, y de entre los asiáticos destacan también las ganancias de peso de India (0,8 puntos) y Vietnam (0,7 puntos). Por el contrario, el peso de las importaciones japonesas se redujo en 1,5 puntos porcentuales.

\subsection{Comercio de servicios de España}

Según los datos del Banco de España, Asia en su conjunto, incluyendo Oriente Próximo y Medio, representaba en 2016 el 8,3 por 100 de los ingresos y el 6,7 por 100 de los pagos, porcentajes que disminuyen, si se excluye Oriente Próximo y Medio, a 3,9 por 100 para ingresos y a 5,3 por 100 para pagos, con un superávit a favor de España de 1.161 millones de euros. China y Japón son los principales socios de Asia, con el 0,8 y 0,7 por 100, respectivamente, de los ingresos y el 2,0 por 100 y el 0,6 por 100 de los pagos.

China es el segundo país importador de servicios comerciales, con el 9,6 por 100 del total mundial en 2016, y registra el mayor déficit comercial, con más de 240.000 millones de euros. Entre los veinte principales países de importación se encuentran también otros asiáticos: Singapur, India y Corea del Sur. Sin embargo, China es el país con el que España registra el mayor déficit en servicios: 315 millones de euros.

Como el Banco de España no proporciona datos de Oriente Próximo y Medio, para poder diferenciar entre los ingresos por servicios turísticos y no turísticos, los comentarios corresponden a Asia en su conjunto. Respecto a los servicios turísticos, en 2016 los ingresos alcanzaron los 54.660 millones de euros, el 47,7 por 100 del total de los servicios, y Asia supuso solamente el 4,1 por 100. Por ello, parece oportuno intensificar la labor para la diversificación de los mercados emisores, en especial para captación de mercados más alejados, entre ellos los asiáticos, y de alto potencial de crecimiento, como China, Japón e India.

La diversificación geográfica de las exportaciones de servicios no turísticos es superior a la de los servicios turísticos y a la del comercio de bienes. Nuevamente, Asia, incorporando los países de Oriente Medio, se sitúa en tercer lugar, con el 12,1 por 100 de los ingresos, por detrás de América (21,3 por 100), y es el área donde más están creciendo las exportaciones españolas de este tipo de servicios. En cuanto a la evolución entre 2013 y 2016, destaca la ganancia en peso de Asia (2,8 puntos porcentuales), siendo el mercado más dinámico, con un crecimiento medio anual del 17,6 por 100 .

Además de la importancia de China en el continente asiático por su tamaño (1.378 millones de consumidores y una renta per cápita de 8.260 USD en 2016, casi el doble que en 2010) y sus perspectivas a medio plazo (crecimiento del 6,6 y 6,4 por 100 para 2018 y 2019 respectivamente, según la OCDE), me gustaría también mencionar el Plan Made in China 2025, que es una iniciativa del Gobierno chino, $\triangleright$ 
cuyo objetivo es continuar transformando el país hacia una potencia tecnológica. Para ello se establecen medidas para impulsar la restructuración y el desarrollo del sector industrial y así alcanzar una mayor calidad y eficiencia en la producción, haciendo que la industria manufacturera «ascienda» en la cadena de valor.

Esta estrategia de desarrollo se enmarca dentro de un programa de treinta años de duración, que se ejecutará en tres fases: primera, reducir las diferencias con otros países (2025); segunda, fortalecer la posición (2035); y tercera, liderar la innovación (2045). Para conseguir estos objetivos, el Gobierno chino aplicará políticas favorables a la reestructuración de la industria tradicional manufacturera, haciendo especial énfasis en la innovación, la propiedad intelectual y el desarrollo sostenible, al mismo tiempo que se promueve la fusión y la reorganización de las empresas.

El Plan Made in China 2025 difiere de anteriores planes para la industria china promulgados por el Gobierno, ya que el enfoque de este no se centra solo en la innovación, sino en el proceso de fabricación completo, tanto de la industria más novedosa como de la tradicional. Además, se dota de mayor protagonismo a los mecanismos de mercado, sin olvidar la intervención estatal.

Este plan representa nuevas oportunidades para las empresas extranjeras, ya que China necesitará la tecnología, el know-how y el personal cualificado necesarios para poner en marcha dicho proyecto.

\section{El Plan China}

El Plan China está estructurado en tres grandes apartados. El primero recoge las relaciones económicas y comerciales actuales entre China y España. Se cuantifican las relaciones comerciales de bienes y servicios, las inversiones españolas en China y viceversa, así como el número de empresas españolas que exportan a China en total, con carácter regular y las que se han establecido en el país. El segundo apartado está dedicado al plan propiamente dicho. Se estructura teniendo en cuenta los seis ejes estratégicos y los objetivos del EIEE 2017-2027, pero centrándose en las actividades que más afectan a los sectores seleccionados como prioritarios respecto de China. También se recoge el presupuesto de gastos corrientes y recursos humanos disponibles de la Secretaría de Estado de Comercio para China. El tercer apartado es el dedicado al seguimiento y evaluación del plan. Es muy importante, pues los planes tienen que ser lo suficientemente flexibles para incorporar los cambios que se vayan produciendo y se consideren necesarios introducir a la luz de los resultados de las actividades realizadas.

\subsection{Situación actual de las relaciones económicas y comerciales bilaterales}

\subsubsection{Relaciones comerciales bilaterales}

A pesar de la fortaleza y dinamismo del sector exterior español en China en los últimos años, dichas relaciones se encuentran históricamente desequilibradas. En 2016 se importaron 23.851 millones de euros y se exportaron 5.032 millones de euros. A pesar de que las exportaciones españolas crecieron un 14,8 por 100 , el déficit comercial alcanzó los 18.819 millones de euros, con una tasa de cobertura del 21,1 por 100 (llegó a ser del 10,5 por 100 en 2008).

En 2016, China fue el undécimo cliente de España, con un 2 por 100 del total de la $\triangle$ 
exportación española, y el tercer suministrador, con un 8,7 por 100 del total de las importaciones (principal proveedor de España fuera de la UE). China es el primer destino en Asia de nuestras exportaciones, seguido de Japón y Corea. Los principales sectores de la exportación española son alimentos (22,35 por 100$)$, seminanufacturas $(21,24$ por $100)$, materias primas $(20,23$ por 100$)$, bienes de equipo (16,93 por 100), manufacturas de consumo (10,55 por 100) y sector automóvil $(6,71$ por 100$)$. Destaca que nuestras exportaciones están muy concentradas en pocos capítulos, según el sistema armonizado (SA), los cuatro primeros suponen más de la mitad del total.

La cuota de las importaciones chinas procedentes de España sobre el total de sus importaciones alcanza el 0,4 por 100, pero en bienes de equipo apenas llega al 0,2 por 100 (siendo los bienes de equipo la principal partida de las importaciones totales chinas, con un 42,5 por 100 del total o 675.000 millones de USD en 2016). Por encima de esa media se sitúa el sector de alimentación, bebidas y tabaco, con el 1,3 por 100, bienes de consumo duradero, con el 1,2 por 100, y en menor medida también los productos químicos (0,7 por 100) y el sector automóvil $(0,7$ por 100).

\subsubsection{Inversiones}

En el ámbito de las inversiones también se manifiesta un desequilibrio importante. China ocupa el puesto 10 del ranking de países con inversión en España. En 2016, la inversión bruta española en China fue solo de 122,9 millones de euros, con 2.769 millones de euros de stock en 2015. China ocupa el puesto 24 en el ranking de países como destino de la inversión española, con un 0,64 por 100 del total. China invirtió en España 1.117 millones de euros en 2016 y el stock de inversiones era de 9.206 millones de euros en 2015, el 2,65 por 100 del total.

Hay unas seiscientas empresas españolas con implantación en el mercado chino en los sectores de componentes de automoción, energías renovables, servicios financieros, servicios legales, telecomunicaciones, transporte de pasajeros, confección, turismo (hoteles), ingeniería, química y siderurgia.

En enero de 2017 se reunió en Pekín el Grupo de Trabajo de Inversiones para concretar proyectos para financiarlos juntos y para resolver los problemas que las empresas de ambos países tienen en la realización de sus proyectos de inversión.

\subsubsection{Empresas españolas exportadoras}

En 2016, 15.051 empresas españolas exportaron a China, de las cuales 5.194 lo hacen con carácter regular. Cifras bastante significativas, pero sigue existiendo un gran potencial para incrementarlas y diversificarlas, con un mayor valor de exportación por empresa. En 2016, 9.973 empresas exportaron menos de 5.000 euros; 123 empresas, entre 5 y $50 \mathrm{mi}$ llones; y solamente 13, más de 50 millones de euros. Así, las 10 primeras empresas exportadoras a China suponen casi el 30 por 100 del total; las 100 primeras, el 65 por 100, y las 1.000 primeras, el 93 por 100 .

Sin embargo, mirando los datos con perspectiva, en el periodo 2010-2016, el número de empresas españolas que exportaron a China se multiplicó más de dos veces y duplicó a las que lo hicieron a Japón (7.011) en 2016. En el contexto global, el número de empresas que $\triangleright$ 
exportó a China en 2016 es similar al de las que exportaron a Portugal (15.991) y por encima de las que lo hicieron a otros mercados importantes de la UE. Esto pone de manifiesto que la mayoría de las empresas exportadoras a China lo hacen en cantidades relativamente pequeñas. Esto se puede interpretar como una apuesta a largo plazo, al haber conseguido las empresas españolas «poner un pie» en el mercado chino.

\subsection{Estructura: actividades del Plan China}

El Plan China se ha estructurado teniendo en cuenta la EIEE 2017-2027 y el Plan de Acción 2017-2018, es decir, se consideran sus seis ejes estratégicos y sus objetivos, que van a definir tanto las actuaciones como las actividades. Para el Plan China, tanto las actuaciones como las actividades están centradas en los sectores elegidos como estratégicos. Para la selección de estos sectores han sido clave los insumos que nos han suministrado las Ofecomes que tenemos en China (Pekín, Shanghai, Hong Kong y Cantón) y el ICEX. Estas oficinas no solamente han ayudado a identificar estos sectores, sino que van a ser líderes en la preparación y ejecución de algunas de las actuaciones/actividades previstas para los próximos años.

A continuación voy a mencionar los sectores prioritarios para la exportación española a China establecidos en el plan, recordando que no excluyen otras actividades previstas para otros sectores: tecnología industrial (destacan la automoción y la aeronáutica), Industria 4.0 (destacan automatización y robótica, biotecnología, smart cities y startups), sanidad, servicios educativos, sector agroalimentario (entre otros productos, jamón, vino, aceite, frutas, carne fresca y conservas) y turismo y bienes de lujo.

Para todos estos sectores prioritarios se van a utilizar todos los instrumentos oficiales de apoyo a la internacionalización, pero especialmente todos los que tienen que ver con la promoción comercial y que están a cargo del ICEX (ferias, congresos, seminarios, foros, misiones directas e inversas, jornadas técnicas...). Pues bien, la previsión del ICEX para el año 2018 es realizar numerosas actividades de promoción relacionadas con el mercado chino, incluyendo las que se realizarán en España, y también se incluyen las actividades realizadas con la atracción de inversiones. No olvidemos que China es el país donde más actividades realiza el ICEX, después de la UE y EEUU.

Además de estas actividades relacionadas más con la promoción (entrarían dentro del primer eje de la EIEE 2017-2027: «ofrecer un apoyo a la internacionalización cada vez más adaptada a las necesidades y al perfil de nuestras empresas»), la Secretaría de Estado de Comercio va a seguir utilizando todos sus instrumentos institucionales y financieros para apoyar la exportación a China y las inversiones (tanto las procedentes de China como las que van dirigidas a España) que han demostrado ser exitosas en los últimos años. En el ámbito financiero, tenemos que recordar que, en las líneas estratégicas del FIEM (Fondo para la Internacionalización de la Empresa), China es un país prioritario. Asimismo, COFIDES tiene una línea china con una dotación anual de 55 millones de euros para financiar proyectos privados viables con interés español que se realicen en China, dando prioridad al sector de infraestructuras y servicios, que incluye: energías renovables y cogeneración, transporte y telecomunicaciones, medioambiente y $\triangleright$ 
tratamiento de residuos, plataformas y servicios logísticos, y potabilización y depuración de aguas.

En el Plan China se quiere reforzar estos instrumentos financieros generales de apoyo a la empresa española: COFIDES reforzará su línea país para China, CESCE explorará la posibilidad de colaborar con SINOSURE en proyectos en los que participen empresas españolas y chinas, y el FIEM tendrá un mayor protagonismo, tanto de su vertiente comercial (se difundirá entre potenciales beneficiarios chinos tanto públicos como privados a través de los bureaux del Ministerio de Finanzas chino) como para apoyar las inversiones de empresas españolas en China (por ejemplo, el Project-finance con garantías suficientes).

Como dijimos en la introducción, el Plan China incorpora algunas iniciativas que le dan un importante valor añadido en relación con lo que se hacía con los PIDM. Me referiré a algunas de ellas teniendo en cuenta los ejes estratégicos de la EIEE 2017-2027.

Con relación al segundo eje, «incorporar la innovación, la tecnología y la digitalización a la internacionalización", siguiendo un enfoque sectorial en el ámbito del e-commerce, se quiere firmar un MoU (Memorandum of Understanding) de ICEX con Alibaba para abrir nuevas vías en el comercio electrónico de productos españoles hacia China, completando los MoU ya firmados con JD.com y DH Gate. También se quiere valorar la conveniencia de firmar un MoU con VIP.com, plataforma especializada en el comercio electrónico de bienes de consumo de lujo.

En relación con el tercer eje, «desarrollar el capital humano para la internacionalización», se van a explorar las oportunidades de colaborar con otras instituciones $u$ organismos chinos para desarrollar un programa de postformación de los becarios ICEX. En este sentido, se quiere realizar un programa de becarios en el China Development Bank.

En relación con el cuarto eje, «aprovechar las oportunidades de negocio de la Política Comercial Común y de las Instituciones Financieras y Organismos Multilaterales", se van a llevar a cabo acciones en Bruselas y con la delegación de la UE en Pekín que permitan avanzar en el Acuerdo de Inversiones de la UE con China, asegurándonos una consolidación de la apertura a la inversión en el mercado chino, pero tratando de conseguir ventajas preferenciales de acceso a ese mercado.

También se va a dar un impulso para una pronta conclusión de las negociaciones para la renovación de un Acuerdo de Doble Imposición que no discrimine a las empresas españolas frente a las europeas.

Dado que ya ha sido ratificado y va a entrar en vigor el 1 de marzo de 2018, se va a dar difusión a las ventajas del acuerdo bilateral en Seguridad Social entre ambos países, el cual es importante a la hora de reducir costes de las inversiones españolas en China.

Una de las prioridades de este eje será la firma de un MoU con MOFCOM (Ministerio de Comercio de China) de cooperación en terceros mercados, con la creación de un grupo de trabajo a nivel de director general que permita crear una plataforma institucional en la que invitar a actores empresariales y financieros para identificar y dar seguimiento a los proyectos que se desarrollen a lo largo de la Ruta de la Seda y también en otras regiones. Precisamente, se quiere firmar un $\mathrm{MoU}$ de colaboración con el China Development Bank para cofinanciar e impulsar proyectos entre empresas españolas y chinas en terceros mercados, en especial a lo largo de los países de la Ruta de la Seda.

En este eje, tampoco podemos olvidar la necesidad de coordinarnos con la Delegación $\triangleright$ 
de la UE en China en las acciones de la llamada «diplomacia económica europea», que incluyen tanto las acciones de promoción como las de «acceso a mercados».

Con relación al quinto eje, «potenciar la captación y consolidación de la inversión extranjera de alto valor añadido", Invest in Spain aprobó unas directrices de actuación de las Ofecomes en materia de atracción de las mismas y sobre el posicionamiento de España como plataforma global de negocios e inversiones para Europa, Oriente Medio-África y Latinoamérica. Para China, como uno de los países prioritarios, se han establecido tres actuaciones y nueve actividades a realizar por sus Ofecomes para potenciar la capacidad de España de atraer inversiones extranjeras de China. Asimismo, se prevé la celebración del Grupo de Trabajo de Inversiones hispano-chino en Madrid en el primer semestre de 2018 para resolver los problemas de la inversión española en China, poniendo el acento en el sector de servicios, y en particular en el financiero.

En cuanto al sexto y último eje de la EIEE 2017-2027, «reforzar la coordinación y complementariedad de acciones entre todos los actores relevantes en materia de internacionalización», es fundamental seguir las políticas de otros países europeos que enmarcan las relaciones económicas con China a un alto nivel político y donde se implican a todos los departamentos ministeriales implicados. En ese sentido, se va a proponer la creación de un grupo de trabajo sobre China dentro del Grupo de Trabajo Interministerial sobre apoyo a la internacionalización de la empresa española, que suponga una coordinación entre todos los ministerios para analizar nuestro grado de colaboración con los homólogos chinos, analizando nuevas iniciativas y realizando una propuesta de refuerzo institucional a la Oficina Económica del Presidente del Gobierno.
También se va a proponer la creación de un grupo de trabajo sobre China en el Consejo Interterritorial de Internacionalización. En una reunión anual con las CCAA se fijarían las actividades que se van a realizar y se haría un repaso de las ya realizadas, con el objetivo de unificar criterios y coordinar viajes oficiales y actividades, teniendo en cuenta los sectores fijados como prioritarios en el PASE China, tanto para la promoción comercial como para la atracción de inversiones.

En este eje no nos podemos olvidar del sector privado, destacando a la CEOE, la Cámara de España, el Club de Exportadores, las Asociaciones de Exportadores, el Foro de Marcas Renombradas y otros organismos. Estas instituciones serán fundamentales para diseñar las actuaciones/actividades del Plan China, así como para la ejecución de una gran parte de las mismas.

El Plan China también cuenta con un presupuesto para el presente año, en el que se señalan los recursos económico-financieros y humanos para llevar a cabo dicho plan. Son los presupuestos de Ofecomes, ICEX, Invest in Spain, las líneas de COFIDES, las del FIEM, ICO, CESCE, etcétera. En el momento de escribir este artículo, no sabemos con exactitud el monto total del dicho presupuesto, ya que todavía hay algunas actividades pendientes de ser confirmadas.

\subsection{Seguimiento y evaluación del Plan China}

Un aspecto muy importante del plan será el seguimiento anual que del mismo se hará y las responsabilidades de cada uno de los centros administrativos en la ejecución del mismo. La experiencia acumulada permitirá ir perfeccionando los planes para años sucesivos. 
Los indicadores para comprobar el grado de cumplimiento de los objetivos señalados en el Plan de China se basarán en algunos que ya están recogidos en el Plan de Acción 2017-2018 y otros que medirán el grado de ejecución de las actividades previstas por parte de las Administraciones Públicas españolas.

\section{Conclusión}

La puesta en marcha del Plan China, así como de los restantes planes PASE, es un reto que la Secretaría de Estado de Comercio considera fundamental para la consecución del principal objetivo que se persigue: consolidar, incrementar y diversificar la presencia de empresas españolas en China, especialmente de las pymes.

El tamaño del mercado asiático y su potencial de crecimiento, unido a la lejanía geográfica, cultural y de marco normativo, que dificultan la presencia española, justifican un mayor apoyo público para facilitar el acceso a los exportadores e inversores españoles de bienes y servicios y, en especial, a su principal destino, China. 